\title{
EFFECTS OF LUMBAR MICRODISCECTOMY ON SPINOPELVIC PARAMETERS
}

\author{
๑ Ümit Ali Malçok, ๑ Ali Akar \\ Çanakkale Onsekiz Mart University Faculty of Medicine, Department of Neurosurgery, Çanakkale, Turkey
}

\begin{abstract}
Objective: One of the causes which accelerates spinal degeneration process is lumbar disc hernia (LDH). In this study, we observed the changes in spinopelvic parameters one month after single-level lumbar microdiscectomy (LM).

Materials and Methods: Standardized bilateral standing scoliosis radiography images of 19 patients, which were performed before and 1 month after LM operation, were evaluated retrospectively. Sagittal vertical axis and coronal vertical axis distances, thoracic kyphosis, lumbar lordosis, pelvic incidence, sacral slope and pelvic tilt angles were measured with Surgimap software.

Results: There was a positive correlation between pre-and post-LM values of all spinopelvic parameters included in the study. In comparative tests however, the difference was found to be not statistically significant. As a result, despite LM operation slightly improved spinopelvic parameters in the first month, there was not a significant change overall.

Conclusion: LDH is known to worsen the degenerative process in spine. LM as a treatment option is seen to alleviate this degenerative process by slowing down the deterioration in spinopelvic parameters.

Keywords: Lumbar microdiscectomy, spinopelvic parameters, spinal balance
\end{abstract}

\section{INTRODUCTION}

Back pain, which has an incidence of $80 \%$ in the general population, is the most common complaint amongst patients who received a lumbar disc herniation (LDH) diagnosis ${ }^{(1,2)}$. The most common symptom after the pain is neurological losses due to radiculopathy. For every 100,000 patients diagnosed with lumbar disc disease, an average of 14-70 lumbar microdiscectomies (LM) are performed ${ }^{(3)}$. The pain can be either directly discogenic, caused by straining of local anatomical structures or paraspinal muscle spasms, and this situation results in a significant productivity loss ${ }^{(4)}$. Besides the spasm of muscles forming lumbar anatomical area, intervertebral disc degeneration and degeneration in facet joints are also shown to affect spinopelvic parameters and cause scoliosis ${ }^{(5)}$. This deterioration in spinopelvic parameters also results in straining of muscles which are responsible for standing straight and ultimately causes pain ${ }^{(6)}$. In another study, it was shown that spinopelvic parameters were deteriorated in these patients ${ }^{(7)}$. It is known that the severity of pain lessens after the correction of spinopelvic parameters ${ }^{(2,8)}$. Deterioration in spinopelvic parameters makes it difficult for patients to live a healthy life and reduces their quality of life ${ }^{(9)}$. Inpatient that underwent instrumentation, the changes in these parameters were studied extensively, and it was found that patients, who were brought closer to the global balance due to these changes, had an increased quality of life $\mathrm{l}^{(10-13)}$.

However, it is seen that the number of studies, which show the changes in these spinopelvic parameters in patients who underwent LM, is fairly low in the literature ${ }^{(11)}$. For this reason, it was aimed to define possible changes in spinopelvic parameters by comparing LM measurement before and after LM in our study. The sagittal vertical axis (SVA), coronal vertical axis (CVA) measurement and lumbar lordosis (LL), thoracal kyphosis (TK), pelvic incidence (PI), pelvic tilt (PT) and sacral slope (SS) angles, which were shown to affect standing posture and global balance ${ }^{(9,12)}$, were included in the study. Surgimap program was used in the measurement of angles and distances ${ }^{(14)}$.

\section{MATERIAL AND METHODS}

Patients who underwent LM operation in Çanakkale Onsekiz Mart University, Department of Neurosurgery, between 01.10.2017 and 1.11.2020 were included in our study. Ethical board approval was received with the (11.11.2020/202013) decision of Çanakkale Onsekiz Mart University, Faculty of Medicine, Ethical Board of Clinical Studies. Permission to use 
medical records for clinical studies were present on the routine patient consent forms received before the operation. Patient data used in the study were analyzed retrospectively. It was shown in the literature that the measurement of spinopelvic angles gave the ideal results when made on simultaneous bilateral scoliosis radiographs which include the area between cranium and femur ${ }^{(15)}$.

Data from 19 cases, whose radiographs were taken in adequate resolution with the proper format for standard angle and parameter measurements, were used in our study. Additionally, cases with radiographs from before and 1 month after the LM operation as routine follow-ups, were chosen. Radiographs which include spinal and pelvic anatomy in proper format were taken from picture archiving and communication system anonymously. Anatomical landmarks, which were used in the measurement of spinopelvic parameters, were determined according to standardized studies ${ }^{(16,17)}$. Parameters PI, PT, SS were used as pelvic parameters and LL, PI-LL mismatch, TK in T4-12 level were used in the thoracolumbar measurement. And for the global alignment parameters, SVA and CVA were used (Figure 1).

For the measurements, Surgimap software (Surgimap, Nemaris, New York, 133 USA) was used ${ }^{(14)}$. The software is distributed for free on the internet. Images of the first cervical vertebra, head of femur and sacrum were inserted to the software before the measurement process (Figure 2). Measurement of spinopelvic
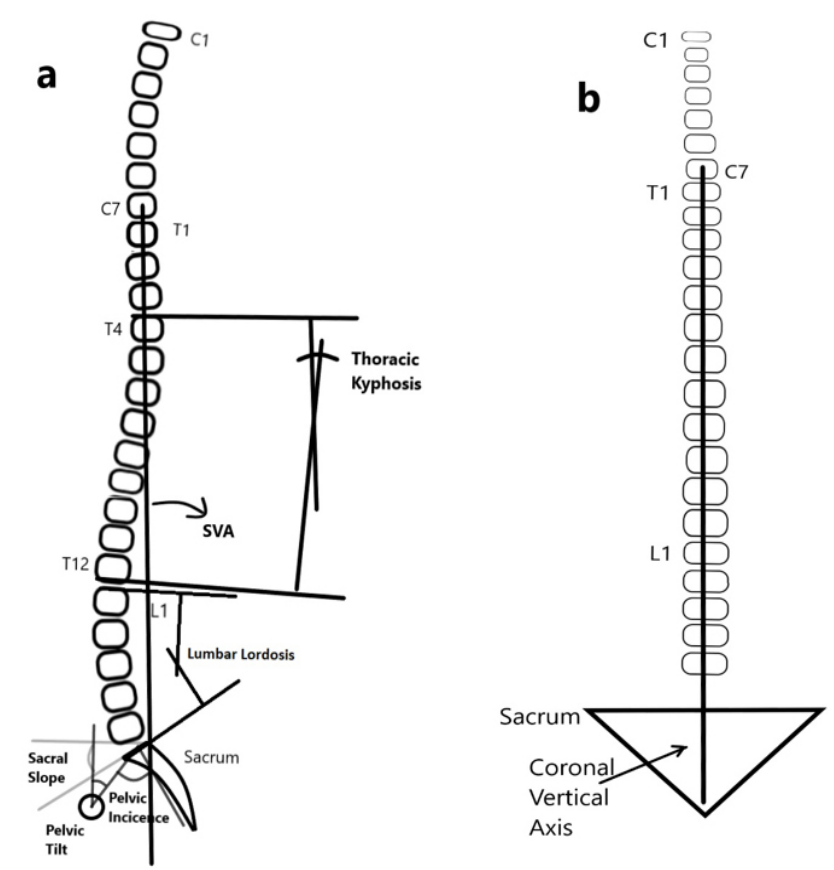

Figure 1. Schematical drawings of the anatomical landmarks that were used a reference for spinopelvic parameters. a) Sagittal plane. b) Coronal plane.
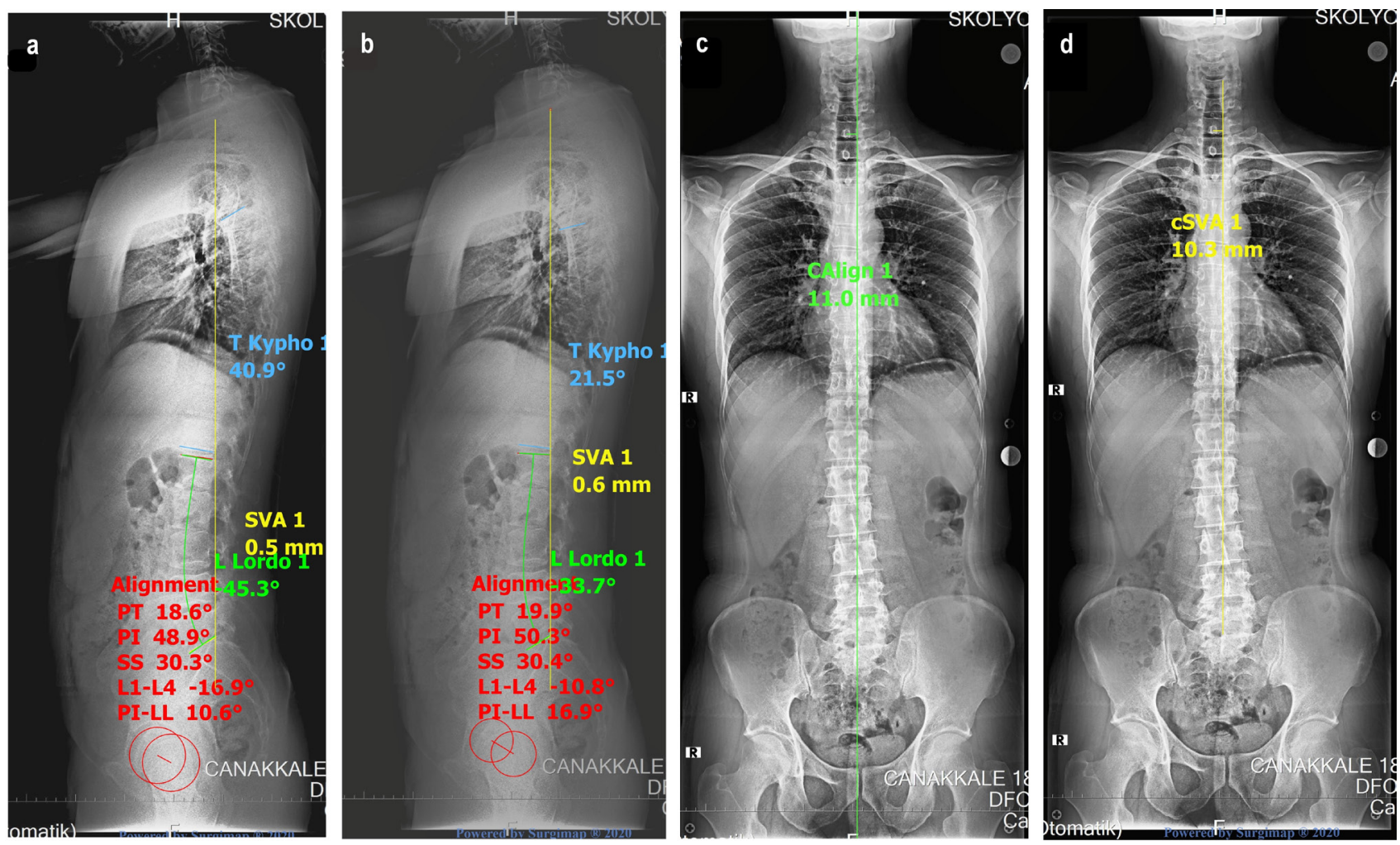

Figure 2. Spinopelvic parameters and measurement techniques. Standing scoliosis radiographs are on a) Pre-operative sagittal, b) Postoperative sagittal, c) Pre-operative coronal, d) Post-operative coronal plane.

SVA: Sagittal vertical axis, CVA: Coronal vertical axis, TK: Thoracic kyphosis, PT: Pelvic tilt, SS: Sacral slope, LL: Lumbar lordosis, PI: Pelvic incidence 
parameters was performed as described in Surgimap user guide ${ }^{(18)}$. Those whose radiographs were not adequate for measurements, who had de-novo or congenital scoliosis, lower extremity asymmetry, congenital hip dislocation, pelvic imbalance, gait deformities, loss of function in peripheral nerves of the lower extremity, advanced lumbar spondylolisthesis, spinal tumour or infection in the lumbar area were omitted from the study. Measurement made on pre-and post-operative radiographs were interpreted by analyzing with comparison and correlation tests.

\section{Statistical Analysis}

Data acquired from patients were transferred to SPSS. First, lost data inspection was made. It was determined that there was no lost data. Pre-and post-operative "SVA, LL, TK, CVA, PT, PI and SS" data of 19 cases were tested for normal distribution through "Shapiro-Wilk test", and it was seen that the data did not have a normal distribution. Bivariate two-sided Spearman correlation coefficient test and Partial two-sided test were used to analyze the existence and direction of correlation. The correlation value (r) calculated between two variables was determined as 0.01 0.29=very weak, $0.20-0.39=$ weak, $0.40-0.59=$ moderate, 0.60 $0.79=$ high and $0.80-1=$ very high correlation. For " $r$ " value, $(+)$ shows positive and (-) shows a negative correlation. Comparative statistical analysis was performed with the Wilcoxon paired two-sample test. The statistical significance threshold was determined as $p<0.05$.

\section{RESULTS}

Nineteen cases that were adequate for spinopelvic parameters were comprised of 11 females (\%57.8) and 8 males (\%42.1). In terms of age, it was seen that there were 5 cases between the ages of 20-40, 9 cases between $41-60$, and 5 cases above the

age of 61 . The mean age was 49.5 for females and 50.1 for males. It was seen that the $L 4-5$ level $(n=10)$ was operated most frequently. Information about age, gender and disc level of the operated cases was given in Table 1.

Between pre-and post-operative means of all spinal parameters, there was a change towards a neutral balance in all groups with the exception of the SS group (Table 2). There was an average of 1.7 increase in SS angle. Despite this change being in the opposite direction of neutral balance, the change was found to be not statistically significant ( $p>0.05$ ).

Comparative statistical tests were performed to determine whether the difference forming in spinopelvic parameters was related to $L M$ operation. To determine the relation between parameters that could affect each other, bivariate correlation method was used. Moreover, relations between parameter clusters and other parameter were analyzed using Partial correlation method.

Table 1. Demographic spread of cases

\begin{tabular}{lll|}
\hline & $\mathrm{n}=19$ & $\%$ \\
\hline Age & & \\
\hline $20-40$ & 5 & 26.3 \\
\hline $41-60$ & 9 & 47.4 \\
\hline $61-80$ & 5 & 26.3 \\
\hline Gender & & \\
\hline Female & 11 & 57.9 \\
\hline Male & 8 & 42.1 \\
\hline Level & & \\
\hline L3-4 & 2 & 10.5 \\
\hline L4-5 & 10 & 52.6 \\
\hline L5-S1 & 7 & 36.8 \\
\hline
\end{tabular}

Table 2. Pre-and post-operative spinopelvic parameter measurements

\begin{tabular}{llllll}
\hline Variable & & Mean & Minimum & Maximum & SD \pm \\
\hline \multirow{2}{*}{ SVA $(\mathrm{mm})$} & Pre-operative & -5.1 & -115.9 & 56.6 & 41.6 \\
\hline & Post-operative & 9.1 & -60.1 & 109,3 & 34.6 \\
\hline $\mathrm{CVA}(\mathrm{mm})$ & Pre-operative & 10.2 & -25.9 & 54.3 & 16.8 \\
\hline \multirow{2}{*}{$\mathrm{LL}\left({ }^{\circ}\right)$} & Post-operative & 6.7 & -14.0 & 44.3 & 15.0 \\
\hline \multirow{2}{*}{$\mathrm{TK}\left({ }^{\circ}\right)$} & Pre-operative & 51.0 & 26.1 & 74.2 & 13.8 \\
\cline { 2 - 6 } & Post-operative & 52.5 & 29.1 & 70.2 & 12.9 \\
\hline \multirow{2}{*}{$\mathrm{PT}\left({ }^{\circ}\right)$} & Pre-operative & 41.5 & 25.8 & 72.9 & 13.5 \\
\hline \multirow{2}{*}{$\mathrm{PI}\left({ }^{\circ}\right)$} & Post-operative & 40.8 & 0.5 & 63.8 & 17.3 \\
\hline \multirow{2}{*}{$\mathrm{SS}\left({ }^{\circ}\right)$} & Pre-operative & 18.5 & 3.1 & 42.2 & 11.7 \\
\hline & Post-operative & 19.8 & 4.0 & 39.9 & 11.4 \\
\hline
\end{tabular}

Mean, minimum and maximum standard deviation values of the measured parameters $(n=19)$.

SVA: Sagittal vertical axis, CVA: Coronal vertical axis, TK: Thoracic kyphosis LL: Lumbar lordosis, PT: Pelvic tilt, PI: Pelvic incidence, SS: Sacral slope 
It was seen that there was a strong positive relation between pre-operative $(-5.12 \pm 41.59 \mathrm{~mm})$ and post-operative $(9.08 \pm 34.61$ $\mathrm{mm})$ SVA measurements $(r=0.63, p<0.01)$. CVA measurements, which were $10.22 \pm 16.17 \mathrm{~mm}$ before the operation, became $6.71 \pm 15.03 \mathrm{~mm}$ afterwards, and there was no statistically significant relation between two values $(r=0.32, p>0.05)$. There is a moderately strong positive relation between post-LM LL angles and previous angles, which was statistically significant $(r=0.53, p<0.05)$. There was a statistically significant, strong positive relation between TK angles $(r=0.64, p<0.05)$. There was a strong, positive correlation between PT angles $(r=0.74, p<0.001)$. The correlation between PI angles was very strong and positive in a statistically significant way $(r=0.83, p<0.001)$. Correlation between changes in SS angles was positive, moderately strong and statistically significant. In relation analysis of spinopelvic parameter measurements, there was a statistically significant, positive change on all planes except CVA (Table 2, 3). All these statistically significant, positive changes show the correctional and protective effect of spinopelvic parameters on global balance, following LM operation.

The PI correlation was determined as the control reference value. A partial correlation test was applied to post-operative values. When the resulting SVA, CVA, TK, PT, SS and LL values were compared with Bivariate test results, the difference was found to be not significant. Similar results were also acquired when LL correlation was determined as a control reference value (Table 4). LL\&PI and LL correlations were determined as control reference parameters. The partial test was applied to pre-and post-operative values. When the way and $r$ values of SVA, PT and SS correlations were compared with Bivariate and partial correlation test results, the difference was found to be not significant (Table 5).

There was no statistically significant difference between " $r$ " values acquired from direct and indirect correlation tests. It was seen that the post-LM measurements were closer to optimal values. These results showed in a statistically significant way that LM operation was effective in bringing spinopelvic parameters closer to the optimum values.

\section{DISCUSSION}

The idea that spinal posture should be mathematically and statistically analyzed was first hypothesized by Beck and Killus in $1973^{(19)}$. Later in 1989, Duval-Beaupere described the economy cone concept, where minimal energy is spent while standing ${ }^{(20)}$. The same author has defined global balance as the position of center of gravity in relation to coccygeal femoral joints ${ }^{(21)}$. Nowadays, despite surgeons continuing to analyze and use the biomechanical effects of global balance and spinal parameters in surgical planning, a consensus can still not be reached on this subject. Global balance concept was refined by further evaluating all these parameters simultaneously, and the importance of these parameters has increased further due to degenerative scoliosis surgery becoming widespread ${ }^{(8,13)}$. Since

Table 3. Correlation in spinopelvic parameters between pre- and post-LM measurements

\begin{tabular}{llllllll}
\hline$(\mathrm{n}=19)$ & SVA $(\mathrm{mm})$ & CVA $(\mathrm{mm})$ & LL $\left({ }^{\circ}\right)$ & TK $\left({ }^{\circ}\right)$ & PT $\left({ }^{\circ}\right)$ & PI $\left({ }^{\circ}\right)$ & SS $\left({ }^{\circ}\right)$ \\
\hline $\mathbf{p}$ & 0.63 & 0.32 & 0.53 & 0.64 & 0.74 & 0.83 & 0.57 \\
\hline $\mathbf{p}$ & $<0.01$ & $>0.05$ & $<0.05$ & $<0.05$ & $<0.001$ & $<0.001$ & 0.05 \\
\hline
\end{tabular}

Bivariate correlation test analysis of parameter measurements, $(n=19)$.

SVA: Sagittal vertical axis, CVA: Coronal vertical axis, TK: Thoracic kyphosis, LL: Lumbar lordosis, PT: Pelvic tilt, PI: Pelvic incidence, SS: Sacral slope

Table 4. Relation between values when compared with post-operative ones

\begin{tabular}{|c|c|c|c|c|c|c|c|}
\hline \multirow{2}{*}{ SVA (mm) } & & \multicolumn{6}{|c|}{ Post-operative } \\
\hline & & CVA (mm) & TK $\left({ }^{\circ}\right)$ & PT $\left({ }^{\circ}\right)$ & $\operatorname{SS}\left({ }^{\circ}\right)$ & $\operatorname{LL}\left({ }^{\circ}\right)$ & \\
\hline Pre-operative and post-operative & $\mathrm{PI} \& \mathrm{PI}$ & $0.58^{*}$ & 0.08 & $0.61^{* *}$ & $0.67^{* *}$ & $0.67^{* *}$ & $0.7^{* *}$ \\
\hline
\end{tabular}

" $r$ " values of parameter measurements from two-sided Partial correlation test analysis. $n=19,{ }^{*} p<0.05,{ }^{* *} p<0.01$.

SVA: Sagittal vertical axis, CVA: Coronal vertical axis, TK: Thoracic kyphosis, PT: Pelvic tilt, SS: Sacral slope, LL: Lumbar lordosis, PI: Pelvic incidence

Table 5. Relation between correlation of pre-and post-operative measurement groups and different measurement groups

\begin{tabular}{|c|c|c|c|}
\hline Pre-operative and post-operative ${ }^{\#}(n=19)$ & SVA & SS & PT \\
\hline PT\&SS & $0.71^{* *}$ & - & - \\
\hline LL\&PI & $0.71^{* *}$ & $0.56^{*}$ & $0.56^{*}$ \\
\hline PT & - & $0.64^{* *}$ & - \\
\hline
\end{tabular}

In this table, correlations between several spinopelvic parameters are shown. (\#r=Spearman Multiplication of Moments Correlation Coefficent) ${ }^{*} p<0.05$, ${ }^{* *} p<0.01$ values were accepted to be statistically significant.

SVA: Sagittal vertical axis, PT: Pelvic tilt, SS: Sacral slope, LL: Lumbar lordosis, PI: Pelvic incidence 
turkishspine

asymmetrical disc degeneration is responsible for scoliosis, it is expected for spinopelvic parameters to be affected in patients undergoing LM operation ${ }^{(5)}$. In literature, there are too few studies in which changes in spinopelvic parameters following LM are analyzed. To discover new information regarding this subject, in our study, we have analyzed SVA and CVA distance, LL, TK, PI, SS and PT angles, which are claimed to change following LM.

SVA, which shows sagittal balance, passes through the posterosuperior corner of S1 when in a neutral position. A positive value is assigned when it passes through the front of this point, and a negative value is assigned when it passes through the back of this point. SVA distance, which is a valuable determinant of balance even by itself, was seen to change towards neutral axis following LM operation, compared to its pre-operative state. It is expected for this change to lighten degenerative loads on the disc and to slow down the degenerative process. It is considered a neutral global balance when CVA passes through posterosuperior of S1 vertebrae.

CVA is assigned a positive value when it passes through the right side of the patient and a negative value when it passes through the left side (Figure 1b). When a spinal pathology is on multiple levels and is advanced, coronal balance becomes disrupted. We have reached the conclusion that the effect of LM on CVA was not statistically significant in our series. However, the difference between post- $(6.7 \pm 15)$ and pre-LM $(10.2 \pm 16.2)$ CVA values we obtained was slightly in favour of global balance (Table 3).

All loads that are transferred through the spine are transmitted to lower extremities by the pelvis, and the direction of these force vectors change according to pelvic parameters. PI, which is one of these parameters, was shown to not change for the entire life of a person after bone growth was completed, unless the pelvic structure was disrupted by a pathology ${ }^{(9)}$. In a study made by no relation was found between the severity of disc degeneration and PI values in both surgical intervention group and conservative treatment groups $^{(22)}$. Moreover, they also reached the conclusion that there was no statistically significant difference between PI values of patients that underwent $L M$ or instrumented spinal fusion surgery.

It is known that $\mathrm{PI}=\mathrm{PT}+\mathrm{SS}(\text { Figure } 1)^{(9)}$. Since it is not possible for the PI angle to change under normal circumstances, it should be considered that the change might be resulting from the researcher who made the measurements or measurements on non-standard radiographs. In our measurements, the mean 3-degree difference seen in PI angles was thought to be a result of the aforementioned phenomena (Table 2). The strong relation we saw between pre-and post-LM PI angles when they were statistically compared showed that there was no statistically significant difference. On top of this, the strongly positive result from the comparison of pre-and postoperative PT\&SS and LL\&PI relation coefficients with SVA relation coefficients showed that LM operation did not cause a statistically significant change on these angles.
SS and PT measurements, which are part of the pelvic parameters, were seen to have stayed the same. When correlations of different measurement groups were analyzed, it was clear that the relation between $\mathrm{PI}$ and other angles were not disrupted. Moreover, the post-LM change in PT and SS angles was seen to be in favour of global balance. However, in another study, it was determined that pathologies, which caused a decreased lumbar disc distance, changed LL and as an extension, SS and $\mathrm{PT}^{(23)}$. It is known that the flattening in $\mathrm{LL}$ is related to $\mathrm{LDH}$, and this decrease in lordosis is known to increase disc degeneration, and trigger compensation mechanisms such as increased thoracic kyphosis ${ }^{(24)}$. Between pre-and post-operative LL and TK angles of patients who underwent single-level LM, a moderately strong, positive correlation was found (Table 3). Despite a minimal change in angles following discectomy, this difference was found to be not statistically significant after comparative statistical tests ( $p>0.05$ ). To sum up, all changes in spinopelvic parameters were seen to be in favour of global balance following LM.

\section{Study Limitations}

Further studies made on large series which also include sixth month and first-year parameters, not just early post-operative period, are needed to further clarify the relation between spinopelvic parameters and LM. Additionally, studies that include a control group comprised of patients who needed LM operation but were unable to be operated would also be helpful.

\section{CONCLUSION}

One of the pathological elements that play a role in degenerative spinal process is $L D H$, and LM is commonly used as a treatment option. In our study, we aimed to determine whether the spinopelvic parameters changed with LM treatment in LDH cases which required surgical treatment. The data we collected showed that there was a positive correlation between preoperative and post-operative first-month measurement. These results show that spinopelvic parameters are protected, or even improved towards global balance following LM. Moreover, it supports the hypothesis that LM alleviates and slows down the deterioration in spinopelvic parameters related to disc degeneration.

\section{Ethics}

Ethics Committee Approval: Ethical board approval was received with the (11.11.2020/2020-13) decision of Çanakkale Onsekiz Mart University, Faculty of Medicine, Ethical Board of Clinical Studies.

Informed Consent: Permission to use medical records for clinical studies were present on the routine patient consent forms received before the operation.

Peer-review: Internally peer-reviewed. 


\section{Authorship Contributions}

Surgical and Medical Practices: Ü.A.M., A.A., Concept: Ü.A.M., Design: Ü.A.M., Data Collection or Processing: Ü.A.M., Analysis or Interpretation: Ü.A.M., A.A., Literature Search: Ü.A.M., A.A., Writing: Ü.A.M., A.A.

Conflict of Interest: No conflict of interest was declared by the authors.

Financial Disclosure: The authors declared that this study received no financial support.

\section{REFERENCES}

1. Andersson GB. Epidemiological features of chronic low-back pain. Lancet. 1999;354:581-5.

2. Smith JS, Shaffrey Cl, Berven S, Glassman S, Hamill C, Horton W, et al. Improvement of back pain with operative and nonoperative treatment in adults with scoliosis. Neurosurgery. 2009;65:86-93.

3. Lagerbäck T, Fritzell P, Hägg O, Nordvall D, Lønne G, Solberg TK, et al. Effectiveness of surgery for sciatica with disc herniation is not substantially affected by differences in surgical incidences among three countries: results from the Danish, Swedish and Norwegian spine registries. Eur Spine J. 2019;28:2562-71.

4. Petit A, Roquelaure Y. Low back pain, intervertebral disc and occupational diseases. Int J Occup Saf Ergon. 2015;21:15-9.

5. Aebi M. The adult scoliosis. Eur Spine J. 2005;14:925-48.

6. Zhang G, Yang Y, Hai Y, Li J, Xie X, Feng S. Analysis of lumbar sagittal curvature in spinal decompression and fusion for lumbar spinal stenosis patients under roussouly classification. Biomed Res Int. 2020;2020:8078641.

7. Jackson RP, Peterson MD, McManus AC, Hales C. Compensatory spinopelvic balance over the hip axis and better reliability in measuring lordosis to the pelvic radius on standing lateral radiographs of adult volunteers and patients. Spine (Phila Pa 1976). 1998;23:1750-67.

8. Le Huec JC, Thompson W, Mohsinaly Y, Barrey C, Faundez A. Sagittal balance of the spine. Eur Spine J. 2019;28:1889-905.

9. Schwab FJ, Blondel B, Bess S, Hostin R, Shaffrey Cl, Smith JS, et al. Radiographical spinopelvic parameters and disability in the setting of adult spinal deformity: a prospective multi center analysis. Spine (Phila PA 1976). 2013;38:803-12.

10. Dalbayrak S, Öğrenci A, Akar E, Koban O, Yılmaz A, Yılmaz M. Clinical and radiological outcomes after correction of degenerative lumbar scoliosis with dynamic stabilization (with the help of a rigid rod); and describing an alternative technique. J Clin Neurosci. 2020;79:123-8.

11. Glassman SD, Berven S, Bridwell K, Horton W, Dimar JR. Correlation of radiographic parameters and clinical symptoms in adult scoliosis. Spine (Phila Pa 1976). 2005;30:682-8.

12. Mehta VA, Amin A, Omeis I, Gokaslan ZL, Gottfried ON. Implications of spinopelvic alignment for the spine surgeon. Neurosurgery. 2015;76(Suppl 1):42-56.

13. Roussouly P, Pinheiro-Franco JL. Biomechanical analysis of the spino-pelvic organization and adaptation in pathology. Eur Spine J. 2011;20(Suppl 5):609-18.

14. Surgimap, Nemaris, New York, 133 USA. 2019. https://www. surgimap.com.

15. Faro FD, Marks MC, Pawelek J, Newton PO. Evaluation of a functional position for lateral radiograph acquisition in adolescent idiopathic scoliosis. Spine(Phila Pa 1976). 2004;29:2284-9.

16. Lafage R, Ferrero E, Henry JK, Challier V, Diebo B, Liabaud B, et al. Validation of a new computer-assisted tool to measure spino-pelvic parameters. Spine J. 2015;15:2493-502.

17. Malçok ÜA, Yaman O. Lomber dejeneratif skolyoza yaklaşım. Türk Nöroşir Derg. 2018;28:230-7.

18. Surgimap user guide. Surgimap User Guide Version 3.8. 2015:62-91.

19. Beck A, Killus J. Normal posture of spine determined by mathematical and statistical methods. Aerosp Med. 1973;12:1277-81.

20. Scoliosis Research Society 24th Annual Meeting Amsterdam, Holland September 17-22, 1989, Spine. 1990;15:845. Available from: https://journals.Iww.com/spinejournal/Citation/1990/09000/ Scoliosis_Research_Society_24th_Annual_Meeting.1.aspx

21. Duval-Beaupère G, Schmidt C, Cosson P. A Barycentremetric study of the sagittal shape of spine and pelvis: the conditions required for an economic standing position. Ann Biomed Eng. 1992;20:451-62.

22. Otluoğlu GD, Konya D, Toktas ZO. The Influence of Mechanic Factors in Disc Degeneration Disease as a Determinant for Surgical Indication. Neurospine. 2020;17:215-20.

23. Rajnics P, Templier A, Skalli W, Lavaste F, Illes T. The importance of spinopelvic parameters in patients with lumbar disc lesions. Int Orthop. 2002;26:104-8.

24. Endo K, Suzuki H, Tanaka H, Kang Y, Yamamoto K. Sagittal spinal alignment in patients with lumbar disc herniation. Eur Spine J. 2010;19:435-8. 